\title{
Spontaneous Expulsion of Giant Bladder Stone: Case Report
}

\author{
Julia Tan ${ }^{\mathrm{a}, \mathrm{b}}$, Priyanka Singha ${ }^{\mathrm{a}}$, Eugene Huang ${ }^{\mathrm{a}}$, Hao Chuan Han ${ }^{\mathrm{a}}$
}

\begin{abstract}
Urinary stones of up to $5 \mathrm{~mm}$ of diameter are known to pass spontaneously through the urinary tract; whereas those exceeding $7 \mathrm{~mm}$ of diameter almost always require surgical intervention. To the best of our knowledge, the option of expectant management for large stones is rarely considered. We report an unusual case of a woman who spontaneously passed out two large bladder stones measuring 70 and 60 $\mathrm{mm}$ without having significant pain or bleeding. We find this case striking for the fact that giant bladder stones can expel spontaneously without any surgical manipulations.
\end{abstract}

Keywords: Urinary stones; Bladder; Calculus

\section{Introduction}

Massive or giant bladder calculus is a rare clinical entity. Females are generally less commonly affected than males as $95 \%$ of all bladder stones occur in men [1]. Long-standing uterovaginal prolapse, bladder outlet obstruction, and chronic urinary infection are suspected to be the predisposing factors for bladder calculus. In this report, we present a rare case of a postmenopausal woman who passed out giant bladder stones spontaneously.

\section{Case Report}

A 68-year-old postmenopausal woman was referred to our urogynecology clinic for a lump in the vagina. The symptom started 5 years ago and had gotten worse in the last 8 months. She denied any urinary symptoms. She had a total hysterectomy performed 20 years ago and a history of hypertension which was treated with felodipine. On examination, she had

Manuscript submitted May 22, 2017, accepted June 16, 2017

aDepartment of Urogynaecology, KK Women's and Children's Hospital, 100 Bukit Timah Road, Singapore 229899, Singapore

${ }^{\text {b} C o r r e s p o n d i n g ~ A u t h o r: ~ J u l i a ~ T a n, ~ D e p a r t m e n t ~ o f ~ U r o g y n a e c o l o g y, ~ K K ~ W o m-~}$ en's and Children's Hospital, 100 Bukit Timah Road, Singapore 229899, Singapore. Email: Julia.Tan@mohh.com.sg

doi: https://doi.org/10.14740/jmc2850w a grade 4 cystourethrocele, vault prolapse and rectocele. All other systemic examinations were normal.

Urinalysis showed RBC microscopy $>2,000 \mu \mathrm{L}$, WBC microscopy $>2,000 \mu \mathrm{L}$ and epithelial cells of $21 \mu \mathrm{L}$. Laboratory investigations including hemoglobin, white cell count, urea, and creatinine were within normal range. Pelvic ultrasound reported three large curvilinear echogenic structures suggestive of bladder calculi. They measured 6.6, 4.4 and 3.3 $\mathrm{cm}$ in diameter (Figs. 1 and 2).

Ultrasonography of the urinary system showed both kidneys were of normal size and echopattern. No hydronephrosis or solid renal mass were demonstrated. Bladder was not well distended during the study and hence, the calculi were not identified during the imaging. Urodynamic studies could not be performed as the large cystourethrocele and vault prolapse were irreducible and the patient could not tolerate the procedure.

Urine culture sensitivity reported three episodes of infection with Proteus mirabilis and she was treated with courses of oral cephalexin.

She underwent pelvic floor repair and total Prolift for the cystourethrocele and vault prolapse. The operation was uneventful except that patient was noted to have blood stained urine during catheterization at the end of the operation. Postoperative stay was uneventful and she was discharged on the fourth postoperative day.

The postoperative 1-month follow-up was unremarkable and without any urologic complaint. She was referred to see the urologist for the bladder stones but she defaulted followups with the urologist and with our center. She presented again to the clinic 5 years later after passing out two large urinary stones at home. The size of the stones measured 7 and $6 \mathrm{~cm}$ each. She also reported back pain which spontaneously disappeared after passing out the stones. A thorough physical examination showed no abnormal findings. No bleeding was noted in the vagina and urethra. She was offered renal ultrasound and cystoscopy but she refused. The calculus on biochemical examination showed carbonate, calcium, magnesium, ammonium, urate and phosphate. The urine culture grew Proteus mirabilis which was treated with cephalexin (Fig. 3).

\section{Discussion}

Bladder stones comprise $5 \%$ of urinary tract stones [2]. A giant bladder stone is a rare finding in contemporary clinical practice $[3,4]$. Long-standing uterovaginal prolapse and bladder outlet obstruction coupled with chronic infection in women are pre- 


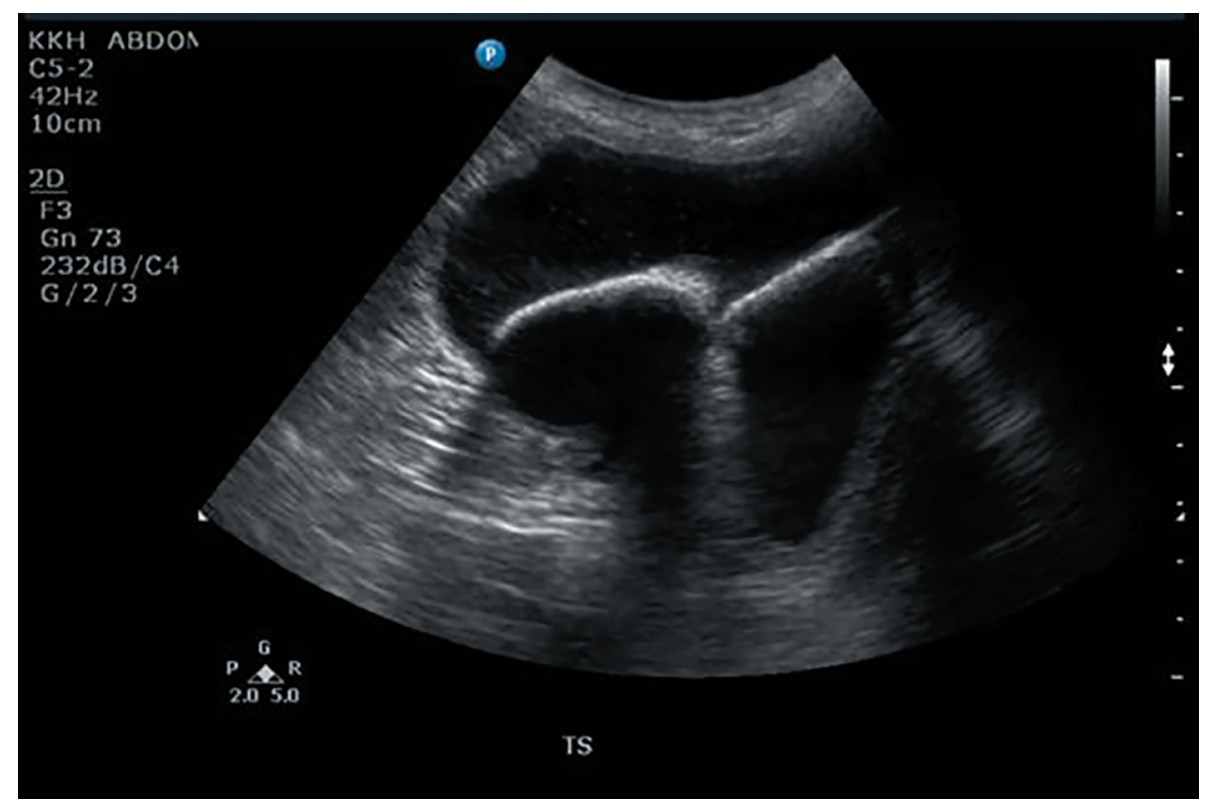

Figure 1. Ultrasound pelvis showing two large bladder calculi.

disposing factors for development of bladder calculi $[5,6]$. In our case, the cystourethrocele and the vault prolapse may have caused incomplete emptying of urinary bladder leading to urinary stasis, recurrent urinary tract infections and providing the environment required for stone development.

The common symptoms of bladder stones are urinary retention, recurrent urinary tract infections and hematuria. Our patient was diagnosed with recurrent Proteus mirabilis infection. This urease-producing uropathogen commonly causes cystitis, acute pyelonephritis and urinary calculus $[7,8]$. Once in the bladder, $P$. mirabilis can form luminal clusters that are the site of bladder stone formation. Bladder stones are also usually observed with renal or ureteral calculi [9], but in our patient, there were no upper urinary tract calculi.

Abdominopelvic planar radiography is commonly used to identify radiopaque stones. Intravenous pyelography (IVP) and computed tomography (CT) can be helpful but the most accurate means of diagnosis is cystoscopy examination [1]. Ultrasonography is also an inexpensive modality to diagnose bladder calculus. The sonogram revealing a classic hyperechoic object with posterior shadowing is effective in detecting bladder stones.

Small stones less than $5 \mathrm{~mm}$ usually pass on their own [10]. In these cases, medication and dietary changes may be all

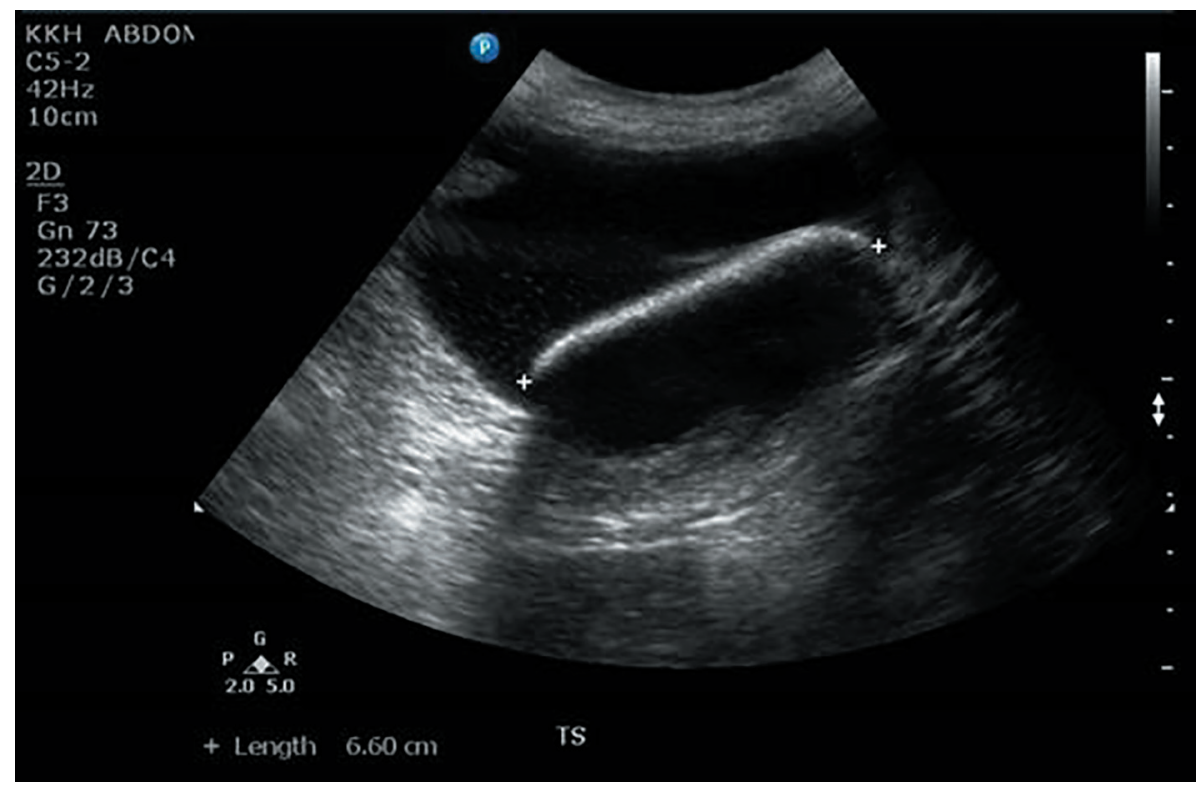

Figure 2. A $6.6 \mathrm{~cm}$ bladder calculus seen on ultrasound pelvis. 


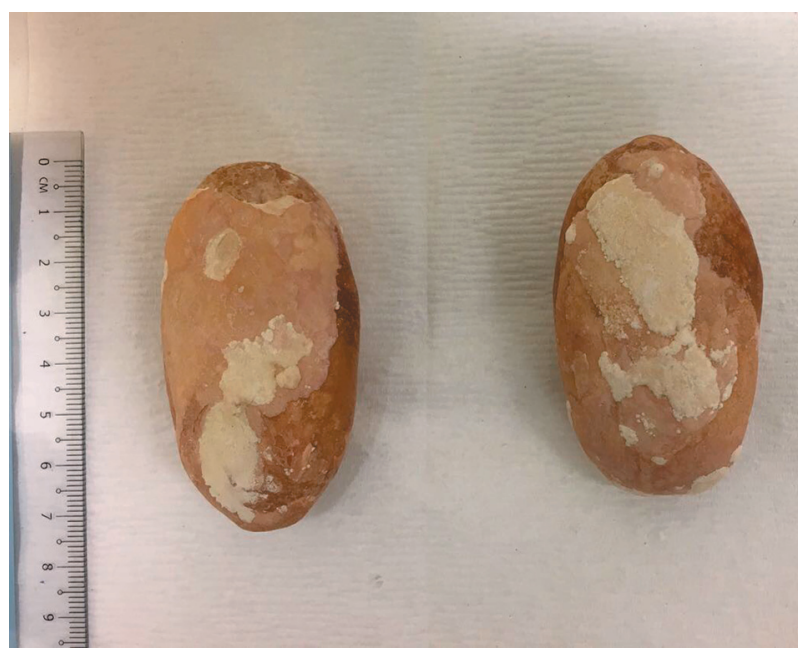

Figure 3. Two giant bladder stones passed out by the patient.

that is needed. Stones of size $5-7 \mathrm{~mm}$ have a modest chance $(50 \%)$ of passage, and those greater than $7 \mathrm{~mm}$ almost always require surgical intervention [11].

Management of large bladder stones consisted of open vesicolithotomy, extracorporeal shock wave lithotripsy and transurethral pneumatic cystolithotripsy [12]. The decision between endoscopic or open cystolithotomy depends on the size and number of stones. If the stones are too large $(>2.5 \mathrm{~cm}$ in diameter) or too numerous to be removed by endoscopic methods, open surgical removal may be necessary [13].

Early diagnosis and early treatment are necessary to prevent long-term pyuria, recurrent infections and renal failure. It should be noted that chronic irritation of the bladder by the stone may ultimately be complicated by bladder carcinoma [14]. Hence, there is the need for long-term follow-up for an early diagnosis of possible bladder carcinoma. In our case, the patient had defaulted her clinic follow-ups and investigations.

\section{Conclusion}

In cases of acute irreducible pelvic organ prolapse, ultrasonography is a relatively inexpensive and rapid modality that can be used to diagnose bladder calculus. Timely follow-ups and appropriate management are necessary in cases of large bladder stones as spontaneous rupture of the bladder to expel the stone can occur, leading to serious complications like intra-abdominal sepsis and vesicovaginal fistula formation. Although spontaneous expulsion of the giant bladder stones in our patient had occurred, open surgical extraction is usually employed and will lead to less morbidity.

\section{Consent}

Informed consent has been obtained from individuals included in this study.

\section{Conflict of Interest}

There is no conflict of interest to declare.

\section{References}

1. Schwartz BF, Stoller ML. The vesical calculus. Urol Clin North Am. 2000;27(2):333-346.

2. Lin WY, Wu CF, Shee JJ, Chen CS. A decade of recurrent cystitis in a woman due to a giant vesical calculus. Int Urogynecol J Pelvic Floor Dysfunct. 2006;17(6):674675.

3. Becher RM, Tolia BM, Newman HR. Giant vesical calculus. JAMA. 1978;239(21):2272-2273.

4. Beisland C, Andersen M. [Giant urinary bladder stone]. Tidsskr Nor Laegeforen. 2000;120(5):573-574.

5. Singal R, Goyal S, Sekhon MS, Pandit S. A rare giant urinary bladder stone: a case report. Urotoday Int $\mathrm{J}$. 2010;3(3).

6. Johnson CG. Giant calculus in the urinary bladder associated with complete uterine prolapse; report of a case. Obstet Gynecol. 1958;11(5):579-580.

7. Rubin RH, Tolkoff-Rubin NE, Cotran RS. Urinary tract infection, pyelonephritis, and reflux nephropathy, in The Kidney, Rector FC, Editor. W.B. Saunders Col.: Philadelphia. 1986. p. 1085-1141.

8. Norsworthy AN, Pearson MM. From Catheter to Kidney Stone: The Uropathogenic Lifestyle of Proteus mirabilis. Trends Microbiol. 2017;25(4):304-315.

9. Aydogdu O, Telli O, Burgu B, Beduk Y. Infravesical obstruction results as giant bladder calculi. Can Urol Assoc J. 2011;5(4):E77-78.

10. Segura JW, Preminger GM, Assimos DG, Dretler SP, Kahn RI, Lingeman JE, Macaluso JN, Jr. Ureteral Stones Clinical Guidelines Panel summary report on the management of ureteral calculi. The American Urological Association. J Urol. 1997;158(5):1915-1921.

11. Zarse CA, McAteer JA, Tann M, Sommer AJ, Kim SC, Paterson RF, Hatt EK, et al. Helical computed tomography accurately reports urinary stone composition using attenuation values: in vitro verification using highresolution micro-computed tomography calibrated to fourier transform infrared microspectroscopy. Urology. 2004;63(5):828-833.

12. Soloway MS. Bladder: excision. In: Hinman Jr F, editor. Atlas of Uro-logic Surgery, vol. 1, 2nd ed. Philadelphia: W.B. Suanders Company; 1998. p. 499-542 [section 13].

13. Torricelli FC, Mazzucchi E, Danilovic A, Coelho RF, Srougi M. Surgical management of bladder stones: literature review. Rev Col Bras Cir. 2013;40(3):227-233.

14. Nygaard E, Terjesen T. Giant vesical calculus and anuria. Scand J UrolNephrol. 1976;10:88-90. 